\title{
Combined genomic and structural analyses of a cultured magnetotactic bacterium reveals its niche adaptation to a dynamic environment
}

Ana Carolina Vieira Araujo ${ }^{1,4}$, Viviana Morillo ${ }^{1,2}$, Jefferson Cypriano ${ }^{1}$, Lia Cardoso Rocha Saraiva Teixeira ${ }^{1}$, Pedro Leão ${ }^{1}$, Sidcley Lyra', Luiz Gonzaga de Almeida ${ }^{3}$, Dennis A. Bazylinski ${ }^{2}$, Ana Tereza Ribeiro de Vasconcellos ${ }^{3}$,

Fernanda Abreu ${ }^{1}$ and Ulysses Lins ${ }^{1 *}$

From 6th SolBio International Conference 2016 (SolBio-IC\&W-2016)

Riviera Maya, Mexico. 22-26 April 2016

\begin{abstract}
Background: Magnetotactic bacteria (MTB) are a unique group of prokaryotes that have a potentially high impact on global geochemical cycling of significant primary elements because of their metabolic plasticity and the ability to biomineralize iron-rich magnetic particles called magnetosomes. Understanding the genetic composition of the few cultivated MTB along with the unique morphological features of this group of bacteria may provide an important framework for discerning their potential biogeochemical roles in natural environments.

Results: Genomic and ultrastructural analyses were combined to characterize the cultivated magnetotactic coccus Magnetofaba australis strain IT-1. Cells of this species synthesize a single chain of elongated, cuboctahedral magnetite $\left(\mathrm{Fe}_{3} \mathrm{O}_{4}\right)$ magnetosomes that cause them to align along magnetic field lines while they swim being propelled by two bundles of flagella at velocities up to $300 \mu \mathrm{m} \mathrm{s}^{-1}$. High-speed microscopy imaging showed the cells move in a straight line rather than in the helical trajectory described for other magnetotactic cocci. Specific genes within the genome of Mf. australis strain IT-1 suggest the strain is capable of nitrogen fixation, sulfur reduction and oxidation, synthesis of intracellular polyphosphate granules and transporting iron with low and high affinity. Mf. australis strain IT-1 and Magnetococcus marinus strain MC-1 are closely related phylogenetically although similarity values between their homologous proteins are not very high.

(Continued on next page)
\end{abstract}

\footnotetext{
*Correspondence: ulins@micro.ufrj.br

1 Instituto de Microbiologia Paulo de Góes, Universidade Federal do Rio de

Janeiro, 21941-902 Rio de Janeiro, RJ, Brazil

Full list of author information is available at the end of the article
} 
(Continued from previous page)

Conclusion: Mf. australis strain IT-1 inhabits a constantly changing environment and its complete genome sequence reveals a great metabolic plasticity to deal with these changes. Aside from its chemoautotrophic and chemoheterotrophic metabolism, genomic data indicate the cells are capable of nitrogen fixation, possess high and low affinity iron transporters, and might be capable of reducing and oxidizing a number of sulfur compounds. The relatively large number of genes encoding transporters as well as chemotaxis receptors in the genome of $\mathrm{Mf}$. australis strain IT-1 combined with its rapid swimming velocities, indicate that cells respond rapidly to environmental changes.

Keywords: Magnetotactic bacteria, Magnetosome, Magnetofaba australis strain IT-1, Biomineralization, mam genes, Genome

Abbreviations: ABC transporter, ATP-binding cassette transporters; BLASTP, Basic local alignment search tool protein; bp, Base pair; CCD Camera, Charge-coupled device camera; CDD, Conserved domain database; CDF, Cation diffusion facilitator; FFT, Fast fourier transform; G + C, Guanidine and cytosine content; GS-GOGAT cycle, Glutamine synthetase-glutamine oxoglutarate aminotransferase cycle; HAADF, High angle annular dark field; KEGG, Kyoto encyclopedia of genes and genomes; mam genes, Magnetosome membrane genes; MCP, Methyl-accepting chemotaxis proteins; mms genes, Magnetosome membrane-specific genes; MTB, Magnetotactic bacteria; mtx genes, Magnetotaxis related genes; NCBI, National center for biotechnology information; ORF, Open reading frame; SMART, Simple modular architecture research tool; STEM, Scanning transmission electron microscope; TCA/ rTCA, Tricarboxylic acids cycle/ reverse tricarboxylic acids cycle; WGS, Whole genome shotgun

\section{Background}

Magnetotactic bacteria (MTB) comprise a morphologically and phylogenetically diverse group of ubiquitous, flagellated, aquatic bacteria capable of orienting along magnetic field lines because of the presence of nanosized intracellular magnetic organelles called magnetosomes [1]. Each magnetosome consists of a nano-sized, membrane-bounded crystal of magnetite $\left(\mathrm{Fe}_{3} \mathrm{O}_{4}\right)$ or greigite $\left(\mathrm{Fe}_{3} \mathrm{~S}_{4}\right)$ usually arranged as (a) chain(s) inside the cell. Magnetotactic cocci have continually been found to be the most abundant morphotype of MTB in freshwater, brackish and marine sediments [2]. While the phenotypic characterization of various magnetococci has been reported, information regarding their ecological, biogeochemical roles in natural environments is limited $[3,4]$. In the magnetotactic cocci, as in other MTB, the magnetotactic response is modulated by chemotaxis (e.g., aerotaxis) in a phenomenon described as magnetoaerotaxis [5]. The magnetic alignment of the cells along the Earth's geomagnetic field lines together with a strong chemotactic response maximizes the efficiency of the organism's search for nutrients and energy (i.e., an optimal position in vertical chemical gradients in the environment) for survival and growth [1].

Genomic data are available for of a number of MTB (Additional file 1). Complete genomes have been described for some Alphaproteobacteria including Magnetospirillum gryphiswaldense strain MSR-1, Magnetospirillum magneticum strain AMB-1, Magnetospirillum caucaseum strain SO-1, Magnetospirillum sp. strain MS-1, Magnetococcus marinus strain MC-1 and Magnetospira sp. strain QH-2
[6-11]. For the Deltaproteobacteria, complete genome sequences are available for Desulfovibrio magneticus strain RS-1 [12]. Partial genomic information is also available for other cultured MTB affiliated with the Alphaproteobacteria $[13,14]$ and cultured and uncultured MTB belonging to the Deltaproteobacteria [15-18], Nitrospirae [19, 20], Candidatus Omnitrophica (part of the Planctomycetes-Verrucomicrobia-Chlamydiae (PVC) bacterial superphylum) [19] and possibly to the Latescibacteria [21, 22]. These partial genomic sequences include descriptions of mam genes for characterization of biomineralization processes in MTB except for the Latescibacterial SCGC AAA252-B13 genome which was used for the identification of an uncultured environmental microorganism [21] and for the detection of mam gene homology without characterization of morphological features of this putative MTB [22].

Magnetotaxis in MTB from both Hemispheres has been characterized [1]. In the Northern hemisphere, MTB predominantly swim northward (i.e., Northseeking) while in the Southern hemisphere, the majority of MTB tend to migrate southward (South-seeking). In both situations, the swimming direction is downward presumably to avoid toxic levels of $\mathrm{O}_{2}$ in surface waters and to find and maintain an optimal position in vertical chemical gradients [23]. Recently, a South-seeking strain named Magnetofaba australis strain IT-1 was isolated in pure culture [13]. Mf. australis strain IT-1 is a magnetotactic coccus that biomineralizes elongated cuboctahedral magnetite magnetosomes [13]. Here, we used a comprehensive approach combining both genomic and ultrastructural analyses to characterize its morphology 
and its potential roles in nitrogen, sulfur, iron and phosphate biogeochemical cycling.

\section{Results and discussion}

\section{General genomic description}

The draft genome of $M f$. australis strain IT- 1 has a size of $4,986,701 \mathrm{bp}$, with a coding density of $82.64 \%$ represented by 4,130 loci, with an average length of 1,010 bp. A total of 2,886 loci encode proteins with putative functions, 44 encode tRNAs and 1,194 encode hypothetical proteins (Additional file 2). Although there is no evidence for the presence of extrachromosomal elements such as plasmids, the occurrence of several lengthy repeat regions hindered genome closure. The genome assembly contains 21 contigs, with a coverage of 33X. Gaps between contigs are mostly regions with unresolved repetitions, caused by the high number of transposable elements found in the IT- 1 genome. The $\mathrm{G}+\mathrm{C}$ content is $57.95 \%$, slightly higher than $\mathrm{G}+\mathrm{C}$ content of Mc. marinus strain MC-1 (54.17 \%) and lower than G + $C$ values found in the freshwater magnetotactic spirillar strains Ms. magneticum strain AMB-1 and Ms. gryphiswaldense strain MSR-1 (65.09 and $63.28 \%$, respectively).

According to KEGG functional classification, most predicted ORFs are related to carbohydrate and amino acid metabolism (162 and 165 ORFs, respectively). A high number of ORFs are related to signal transduction, cell motility and membrane transport (156, 132 and 110 ORFs, respectively). As Mc. marinus strain MC-1 is the only magnetotactic coccus with an available complete genomic sequence, it is not surprising that $51.1 \%$ of $M f$. australis strain IT-1 predicted ORFs were more similar to ORFs described in Mc. marinus strain MC-1 than any other MTB. Nevertheless, similarity between many of their homologous gene sequences is relatively low. Other ORFs were more similar to Magnetospirillum magneticum $(1.1 \%)$ and to non-magnetotactic members of the Proteobacteria phylum ( $<1 \%$ ORFs similar to various strains).

\section{Phenotypical characterization of Mf. australis strain IT-1 Ultrastructure and granular inclusions}

Whole-mount transmission electron microscopy (TEM) of cells of $M f$. australis strain IT-1 shows that they have a unique morphology having both a concave and convex surface (Fig. 1a) confirming the "faba" bean morphology of the cells. Each cell contains a single chain of elongated octahedral magnetosomes and intracellular granules described previously [13]. TEM of ultra-thin sections of cells (Fig. 1b) show that the overall cell ultrastructure of $M f$. australis strain IT-1 is consistent with a two-membrane structure typical of Gram-negative bacteria with a turgid periplasmic gel between them. On the surface of the outer membrane a layer of fibrillar material is present and may represent some type of capsule or $\mathrm{S}$ layer (Fig. 1b; arrowheads); a similar structure was reported in uncultured magnetotactic cocci from the Itaipu lagoon, Brazil [24]. The cytoplasm contains a series of uncharacterized structured regions consisting of "pockets" of amorphous, globular electron-dense material interlaced with electron-lucent regions (Fig. 1b; asterisks). We observed a tabular periodic structure parallel to the inner membrane of some cells in close association with the flagella bundles, most likely corresponding to chemoreceptor arrays (Fig. 1b; white arrows), described also in Ms. gryphiswaldense strain MSR-1 [25], Magnetovibrio blakemorei strain MV-1 [26] and other motile non-magnetotactic strains from different bacterial phyla [27].

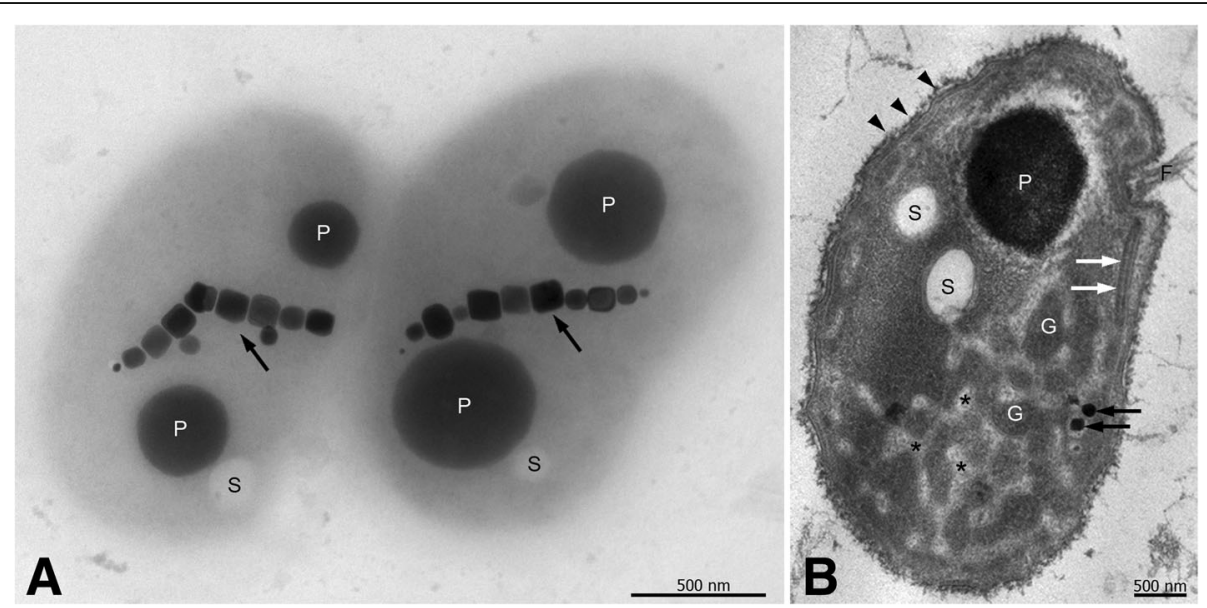

Fig. 1 Ultrastructure of Mf. australis strain IT-1. Ultrastructure of Mf. australis strain IT-1. a Whole mount TEM image showing a single magnetosome chain, P-rich (P) and sulfur (S) granules; (b) Ultrathin section TEM image of high pressure frozen and freeze-substituted cells showing P-rich (P) and sulfur (S) granules, two magnetosomes (black arrows), a flagella bundle (F) associated with chemoreceptor array (white arrows), and a fibrillar layer at the cell surface (arrowheads). Uncharacterized globular structures (G) embedded in an electron-lucent material (asterisks) can be observed 


\section{Flagellar apparatus and motility}

Scanning electron microscopy (SEM) shows that cells possess two bundles of lophotrichous flagella, each at one extremity of the cell (Fig. 2a). These bundles propel cells at swimming speeds up to $300 \mu \mathrm{m} \mathrm{s}^{-1}$. Each flagella bundle consists up to seven separate flagella filaments, with a diameter of $13 \pm 2 \mathrm{~nm}(n=50)$ and a length of $1.9 \pm 0.5 \mu \mathrm{m}(n=20)$. The flagella originate from within a pit located on the cell surface (Fig. 1b). In hanging drop assays under oxic conditions, $M f$. australis strain IT-1 exhibited South-seeking polar magnetotaxis swimming in the presence of the magnetic field of a bar magnet with a fast back and forth swimming pattern near the edge of the drop. A helical trajectory was observed when movement was recorded with a CCD camera using dark-field microscopy (Fig. 2b). However, when analyzed using a high speed camera (500 to $1000 \mathrm{fps}$ ), we observed that over $90 \%$ of $M$ f. australis strain IT-1 cells swim in a straight trajectory by rotating the cell body along an apparent axis parallel to the movement direction (Fig. 2c). This axis is inclined relative to the position of the magnetosome chain which lies along long axis of the cell. The flagella bundles occur at the concave surface of the cell. Surprisingly, Mf. australis strain IT-1 seems to swim with the concave surface forward (Fig. 2c), which strongly suggests that the flagella bundles rotate in front of the cell as the cell body moves.

In the genome of $M f$. australis strain IT-1, genes encoding for every component of the flagellar apparatus (flg, flh, fli; Fig. 2d) are present and those for chemotaxis
(cheA, cheB, cheB/cheR, cheW, motA, MCP to signal translation were also identified. There are approximately 64 genes involved in the synthesis of proteins related to flagellar apparatus and motility. FliPOZNMLWSQREFGI, FlgEKBCG, FlhAB, FlaG, FlgM and 14 copies of a protein containing a flagellar domain, FliC type (ORFs 1035, 1042 to 1044, 1047, 1049, 1059, 2652, 4643 and 5226 to 5230) were detected. These genes form separate groups along the genome (Fig. 2c). Interestingly, the arrangement of the contig containing flgK, fliW, fliD, fliS and $f l i S_{2}$ was similar to the closely related strain MO-1 [28], which is also capable of very high swimming speeds. Flagellin genes share similarity with $\mathrm{fliC}$ genes from magnetococcal strains $\mathrm{MC}-1$ and MO-1, with a slightly higher (1-5\%) similarity with genes from the fast swimming MO-1.

Under aerobic conditions, as previously stated, over $90 \%$ of the cells' motility in Mf. australis strain IT-1 occur in linear rather than helical trajectories and at higher speeds (up to $300 \mu \mathrm{m} \cdot \mathrm{s}^{-1}$, with average of $186 \pm$ $63 \mu \mathrm{m} \mathrm{s}^{-1}$ [13]) than most MTB (average speed ranging from 10 to $120 \mu \mathrm{m} \mathrm{s}^{-1}$ ) $[29,30]$ and similar to that reported in strain MO-1 [31]. The linear trajectory and the magnetosome chain position in the cell raises important issues regarding magnetotaxis as an efficient mechanism for navigation. The magnetosome chain in $M f$. australis strain IT-1 is positioned perpendicular to the axis of movement. Usually, the magnetosome chain is aligned with the axis of cell movement by flagellar propulsion which results in an efficient orientation along the

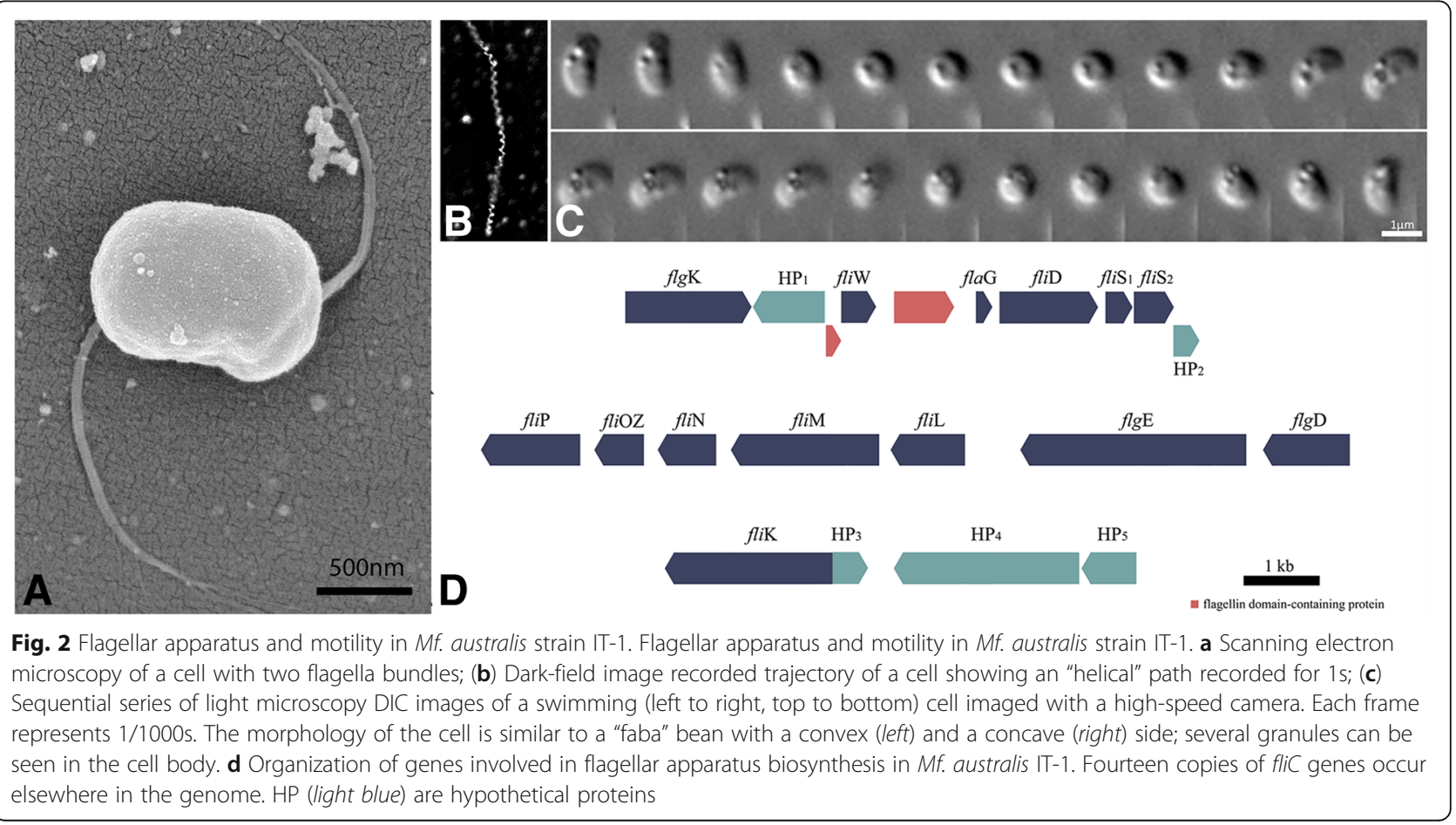


magnetic field [23]. Although magnetosome chains are approximately perpendicular to the swimming axis in Mf. australis strain IT-1, cells orient along magnetic field lines and respond to changes in the magnetic field. Cells of the magnetotactic coccus strain MO- 1 also swim in a straight trajectory, with the magnetosome chain not aligned to the axis of motility [32]. The lack of perfect alignment between the magnetosome chain and the magnetic field lines might be useful for cells to overcome obstacles in their trajectory. The mechanisms for magnetic field orientation and cell dislocation in vertical gradients by flagella propulsion differs from the $\mathrm{t}$ raditional magnetotaxis model described for other MTB $[5,30]$. It is possible that $M f$. autralis strain IT- 1 cells always swim with their flagella in front of the cell body and reversal of swimming direction is achieved by reversing the sense of flagella rotation, as described in other MTB strains [30]. Possibly, MTB described as moving along helical paths is a misinterpretation of results generated by a low speed imaging systems. Interestingly, a flagellar sheath, observed in other magnetotactic cocci or ovoid cells [33], was not observed in Mf. australis strain IT-1. Genes similar to that encoding the Sap protein [34], related to the flagellar bundle sheath in both MO-1 and MC-1 [10], were not found in the IT-1 genome, confirming our microscopy observations and implying that at least for strain IT-1, the sheath is not required for "smooth" swimming as has been suggested [34]. Uncultured cocci from the Itaipu lagoon, when analyzed by freeze-fracture, also did not present a sheath around its bundle of flagella [35]. On the other hand, some cocci showed an intricate arrangement of fibrils that may help to coordinate flagellar movement.

The chemoreceptor arrays, in close association with the flagellar bundles, might allow the cell to control and synchronize the direction and frequency of the rotation of the flagella in the bundle, obviating the need for the flagella sheath that works not only as a protection mechanism but also as a flagella rotation coordinator [34]. The proximity of the chemoreceptor array to the flagellar bundle might allow the cell to respond quickly to environmental changes with greater propulsive force necessary for fast swimming, ensuring cell survival. An MCP-like protein was shown to interact with MamK filaments in Ms. magneticum strain AMB-1 [36], but the similarity values between this protein and the ORFs annotated as MCP in Mf. australis strain IT-1 genome were not high enough to assign this function with certainty. However, due to the high plasticity of sensory domains, it is possible that a MCP whose ligand-binding domain is not described yet carries the coupling between chemotactic sensor and the magnetosome chain in $M f$. australis strain IT-1. A very efficient locomotion system might have evolved in magnetotactic cocci that allowed them to move at high speeds to niches with suitable chemical gradients for their survival. This would make easier to respond to sudden changes in the vertical gradients that may occur in aquatic environments. With the exception of the marine Magnetospira sp. strain QH-2 [11], magnetotactic species with their genome sequenced have a large number of MCP-related genes, characteristic of motile bacteria, with metabolic versatility and that occupies dynamic environments [37].

\section{Magnetosome crystalline habit and genes}

High resolution TEM images of magnetosome crystals (Fig. 3a-b) were indexed with distances and angles between spots being consistent with cubic magnetite $\left(\mathrm{Fe}_{3} \mathrm{O}_{4}\right)$. Tomographic analysis (Fig. 3c) was used to generate an idealized 3D model (Fig. 3d) of octahedral crystal habit elongated along [111] crystallographic direction. Magnetite crystals from magnetosome were isolated from cells growing under heterotrophic conditions with acetate as the carbon source, and averaged $90.42 \pm 19.62 \mathrm{~nm}$ in size with an average shape factor of 0.74 . These values are similar to those reported in other alphaproteobacterial MTB [38] and, although the crystal size is similar to that reported in strain $\mathrm{MC}-1$, the shape factor indicates strain IT-1 has more elongated crystals, similar to those described in strains MO-1 and QH-2 [38].

Mf. australis strain IT-1 mam genes were detected in a 72,493 bp contig. Most genes from this region of the genome (a 40,399 bp fragment), particularly the mam genes, were previously described in Morillo et al. [13] and are more similar to homologous genes described in Mc. marinus strain MC-1, except mamC. Comparison of synteny between $M f$. australis strain IT-1 and Mc. marinus strain $\mathrm{MC}-1$ genomic regions containing mam genes is shown in Additional file 3. Besides similarities in mam and $m m s$ gene organization described by Morillo et al. [13], ORFs encoding hypothetical proteins and a protein with a PilZ domain, known for their participation in chemotaxis [39], are in relatively close proximity to the $\operatorname{mam} A B$ gene clusters, a situation similar to that of $M c$. marinus strain MC-1. BLASTP [40] homology search shows that the only protein similar to the predicted protein containing the PilZ domain belongs to Mc. marinus strain MC-1 (99\% coverage, $31 \%$ ID and $49 \%$ positives), suggesting its possible role in magnetotaxis, by regulating speed and direction of flagellar rotation [39]. The first predicted ORFs within this contig encode two transposases (ORFs 04933, 04812) and one resolvase (ORF 04811) with no homology to Mc. marinus strain $\mathrm{MC}-1$. At the end of the contig genes encoding two transposases (ORF 04938; 04945) and integrases (ORF 05502; 05504) were predicted; these were similar to $M c$. marinus strain MC-1 predicted ORFs (except ORF 04945), but were not found within the putative MAI in 

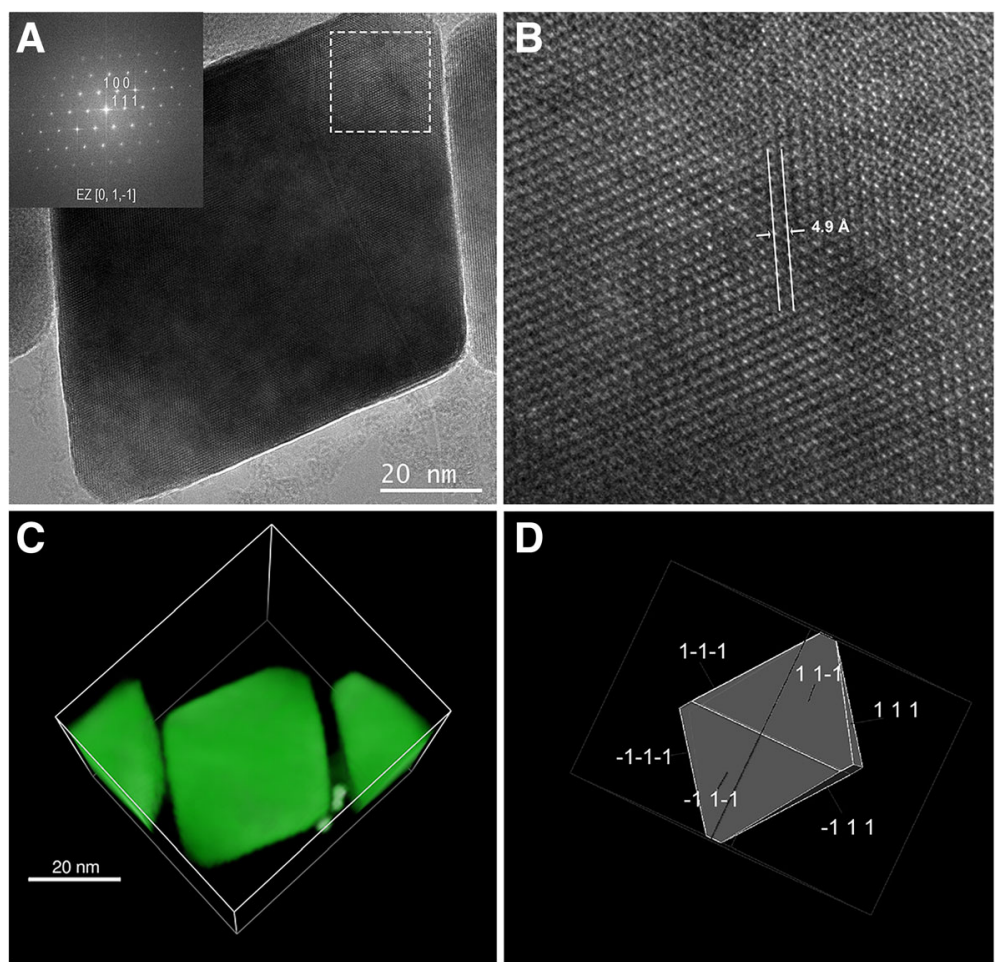

Fig. 3 Magnetosome crystalline habit in Mf. australis strain IT-1. Magnetosome crystalline habit in Mf. australis strain IT-1. a High resolution transmission electron microscopy image of a single magnetosome with elongated octahedral morphology. Inset shows the Fast Fourier Transform with indexed planes and zone axis, (b) Higher magnification image of the dashed boxed are shown in (a), The spacing of fringes shown between white arrows is $4.9 \AA$, consistent with $\left(\begin{array}{llll}1 & 1 & 1\end{array}\right)$ spacing for magnetite. c Tomography reconstruction using STEM/HAADF of the magnetosome shown in (a). $\mathbf{d}$ Idealized model of magnetosome crystal in same orientation shown in (a)

the genome [10]. The identity between the integrases at the end of the contig (ORF 05502 and 05504) is $98 \%$; however, the ORF 05504 represents less than $50 \%$ of the ORF 05502 entire sequence. This contig has 18 predicted hypothetical proteins. Most hypothetical proteins coding ORFs flanking mam genes [13] are only similar to Mc. marinus strain MC-1 predicted hypothetical proteins (identity and positive values vary from 22 to $41 \%$ and 39 to $55 \%$, respectively). Although homology value is not high, the only similar sequence in the database belonged to Mc. marinus strain MC-1 according to BLASP analysis using NCBI non-redundant protein sequence database. The low similarity values for these predicted hypothetical proteins when compared to $M c$. marinus strain $\mathrm{MC}-1$ sequences contrast with those found for a few hypothetical proteins within the mam gene clusters, which identity and positive values range from 22 to $75 \%$ and 36 to $89 \%$, respectively. No results were found for ORFs 02810, 04936 and 02843 based on homology search.

Magnetotaxis related genes ( $m$ tx genes) are localized in a contig of approximately $616 \mathrm{~kb}$ in size. It is not possible to predict its distance from the mam gene cluster, but the contig appears to be part of another cluster, as described for Mc. marinus strain MC-1. This cluster includes three alphaproteobacterial $m t x$ genes, a Sel1 domain-containing protein coding gene, the $m t x A$ gene and an adenylate/guanylate cyclase coding gene. BLASTP best hits were homologous genes described for Mc. marinus strain MC- 1 present in an $m t x$ cluster [10].

\section{Metabolism related genes in Mf. australis strain IT-1}

Figure 4 is an overview of $M f$. australis strain IT- 1 genes showing main the main metabolic pathways and cell components potentially used by this bacterium. Below we describe selected aspects of the genetic information related to biomineralization and metabolism.

\section{Chemoreceptors, transcription and transport}

Magneto-aerotaxis [5] depends on chemoreceptors capable of sensing the external or internal cell conditions. Methyl-accepting chemotaxis proteins (MCPs) are chemotactic sensors coupled to the flagellar apparatus through chemotactic enzymes CheA, CheZ, CheB and CheY, which catalyze (de-)methylation and (de-)phosphorylation reactions that result in the switching of flagella rotation either in the clockwise or anticlockwise direction [41]. According to the conserved topology of 


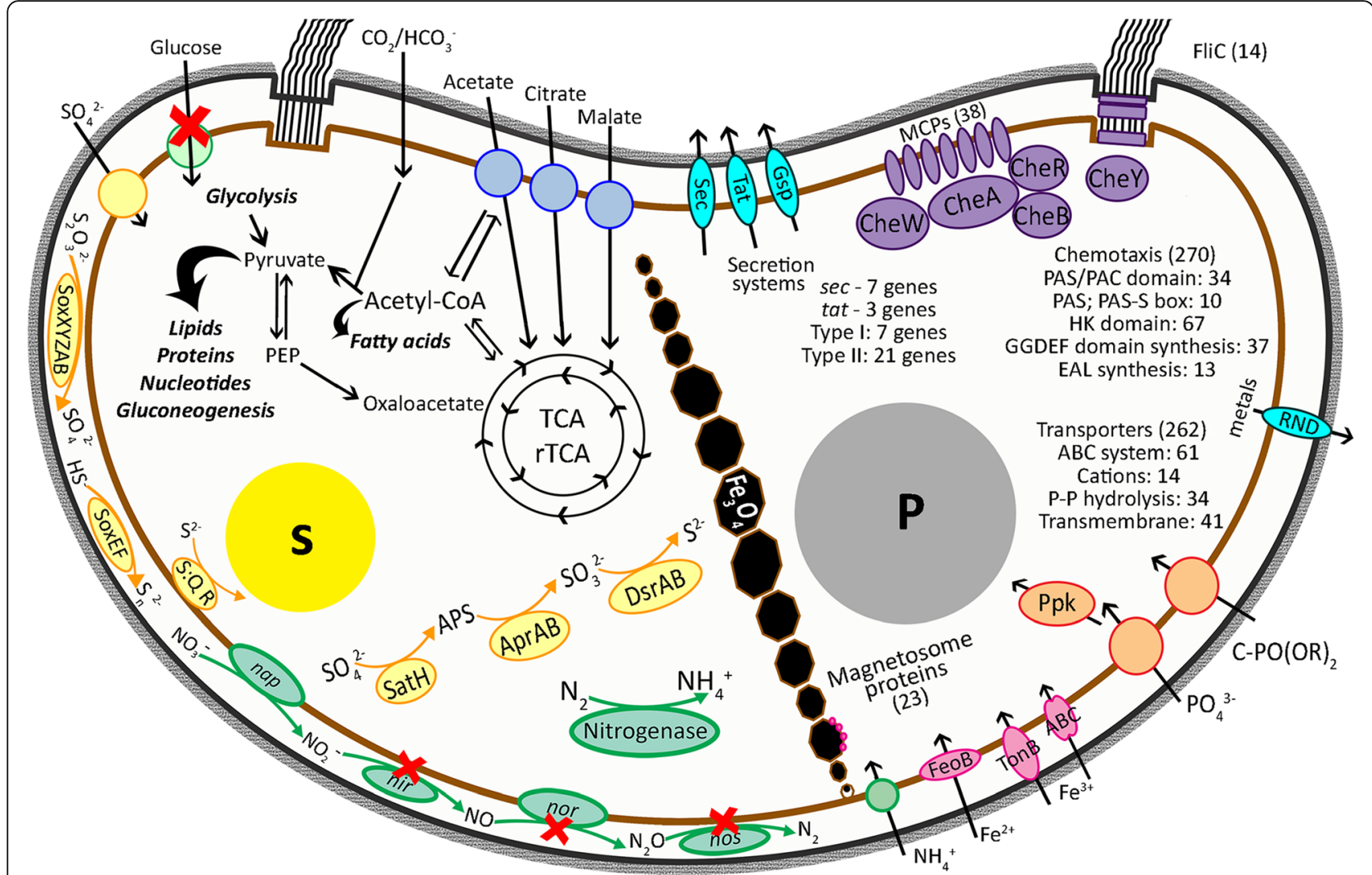

Fig. 4 Schematic overview of Mf. australis strain IT-1 showing its main metabolic pathways and structural features. Schematic overview of Mf. australis strain IT-1 showing its main metabolic pathways and structural features. Cells are bilophotrichous with both flagella bundles in the concave face of the cell. Forty-two genes encode methyl-accepting chemoreceptors usually associated to the flagellar apparatus (purple). Strain IT-1 is chemolitoautotroph using the reverse tricarboxylic acids cycle (rTCA) or chemoorganoheterotroph, capable to grow using small organic molecules such as acetate and citrate. Cells are capable of nitrogen fixation, but probably do not to use nitrate as final electron acceptor (green). Genes for sulfate uptake and reduction were found as well as proteins responsible for sulfur compounds oxidation (yellow) and a gene for a sulfide:quinone reductase (S:Q R), responsible for the synthesis of sulfur globules (S). Genes encoding proteins for the synthesis of polyphosphate granules (P) are present as well as phosphate and phosphanate transporters (orange). High and low affinity iron transporters are encoded (pink) and there are copies of these genes located closely to magnetosome genes, responsible for the synthesis and organization of the chain of magnetite $\left(\mathrm{Fe}_{3} \mathrm{O}_{4}\right)$ cubo-octahedral magnetosomes. Other cell transporters are also depicted (blue)

the domains and their interaction with internal membrane, MCPs have been classified into four main classes [42]. We performed analyses using SMART (Simple Modular Architecture Research Tool) [43] and the CDD (Conserved Domain Database) at NCBI [44] on locus automatically annotated as MCPs. Mf. australis strain IT-1 has 22 MCPs with topology of class I, three resembling a class II topology, 6 similar to class III and 7 type IV MCPs (Additional file 4).

Genomes of MTB generally contain a large number of transcription factor and transport genes presumably enabling them to modulate their magnetotactic behavior in response to diverse environmental stimuli. The large number of transduction components might be used to regulate magnetosome synthesis and magnetotactic behavior [11] and is necessary to avoid unspecific cross talk between different regulatory pathways [45].
The genome of $M f$. australis strain IT-1 contains 272 genes encoding signal transduction proteins (Additional file 5). This number is higher than the number of transduction genes described in the genome of other magnetotactic Alphaproteobacteria strains. Thirty-six transduction genes in $M f$. australis strain IT-1 are related to chemotaxis and includes four genes encoding proteins with CheW domain, three with CheA, three with CheY, two with CheB and three with CheR, two with $\mathrm{CheB} / \mathrm{CheR}$, one with CheX and 13 genes encoding hemerythrin-like metal binding proteins. These hemerythrin-like proteins are involved in oxygen transport but might have domains related to chemotaxis and signal transduction [46].

\section{Iron metabolism}

The first step in magnetite biomineralization is the transport of iron from the extracellular environment into the cell [47]. The genome of Mf. australis strain IT-1 
appears to contain genes necessary for a complete and complex system for capture, transport and regulation of iron including: a large number of iron reductases; $A B C-$ type transporters; ferritins; hemerythrins; and other proteins responsible for iron homeostasis within the cell. All of them are likely important not only for magnetosome biomineralization, but also for cell growth $[48,49]$.

Magnetosome genes in the genome of IT-1 are organized in a contig that also contains genes encoding hypothetical proteins, transposases, integrases, resolvases and an ORF encoding a protein with PilZ domain (ORF 02273). This contig also has ORFs coding hemerythrinlike (ORFs 02806, 02811, 05565), ferritin/ribonucleotide reductase-like (ORF 02816 and 02834), cation diffusion facilitator family transporter (ORF 02835), chromosome partitioning protein (ORF 02842), replication initiator protein A (ORF 02844) and Fis family transcriptional regulator (ORF 04937). For three ORFs encoding hemerythrins, only ORF 02811 has similarity with a gene from Mc. marinus strain MC-1 (35\% identity) whereas two are more similar to predicted proteins from non-MTB (ORF 02806: Treponema brennaborense, 35 \% identity; ORF 02812: Spirochaeta thermophile, 34 \% identity). Because hemerythrin is a protein involved in oxygen transfer, chemotaxis and signal transduction $[46,50]$, the hemerythrin that is common to $M f$. australis strain IT-1 and Mc. marinus strain MC-1, encoded by ORF 02811, is located close to mam genes, and might be involved in magnetotaxis, as suggested [46]. Some ORFs encoding hemerythrin related proteins are also present in other contigs, all related to non-MTB sequences. Ferritin coding ORFs (ORFs 02816, 02834) are homologues to the predicted ferritin-like hypothetical protein in Mc. marinus strain MC-1 (34\% and $47 \%$ identity, respectively). Ferritins are known for their function in iron detoxification, oxidation of $\mathrm{Fe}^{2+}, \mathrm{Fe}^{3+}$ storage and for their controlled release of iron preventing cellular toxicity [51]. If transcribed, ferritins and hemerythrins closely associated with mam genes could be related to magneto-aerotaxis and magnetosome synthesis $[46,51]$, controlling iron redox conditions on the magnetosome crystal or within the magnetosome vesicle, or the oxygen or iron flux. The presence of multiple copies of genes encoding ferritins and hemerythrins in $M f$. australis strain IT-1 suggests their possible role in magnetosome synthesis, as previously described for $f e o A$ and $f e o B$ genes usually present in two copies in MTB genomes [52]. Two copies of the $f e o A B$ system were also found in $M f$. australis strain IT-1 genome (ORFs 02827, 02828, 03818 and 03820). The best blast hits for both copies of $f e o B$ genes of strain IT-1 were sequences of this gene found in other magnetotactic Alphaproteobacteria (99 \% coverage, at least $53 \%$ identity), revealing the high similarity of this gene among MTB. One of the copies, feoB1 (ORF
02827) is located close to mam genes and might be directly involved in magnetosome synthesis whereas $\mathrm{feoB2}$ (ORF 03818) might be involved in cell iron metabolism and detoxification of reactive oxygen species, as proposed in strain MSR-1 [49]. Two Fur (ferric uptake regulator) genes for $\mathrm{Fe}^{3+}$ uptake were also present (ORFs 04154 and 01761), one common to other alphaproteobacterial MTB and other found only in Mc. marinus strain MC-1. When activated by iron or other metal ions, Fur protein binds to the operator of over 90 Furregulated genes in E. coli [53]. The Fur gene from Ms. gryphiswaldense strain MSR-1 has been shown to regulate iron and oxygen metabolism [54], besides having a role in magnetosome formation [48].

The $M f$. australis strain IT-1 genome contains genes that encode iron receptors and transporters of high and low affinity. Genes encoding CDF (cation diffusion facilitator) proteins, also found in Mc. marinus strain MC-1 and other magnetotactic strains are present in the genome, which also contains a gene encoding an iron receptor in outer membrane, $\operatorname{ton} B$, and four additional genes coding TonB family proteins. Besides mam genes involved in redox control and iron stoichiometry ( $m a m E$, $P, T, H, Z$ and $X$ ) during magnetosome formation, a transmembrane protein containing ferric reductase domain (ORF 03272) was found in Mf. australis strain IT-1. This gene has a high similarity to a putative $\mathrm{Fe}^{3+}$ reductase in other MTBs (Ms. magneticum strain AMB1, Ms. gryphiswaldense strain MSR-1, Ms. magnetotacticum strain MS-1, Mc. marinus strain MC-1). Seven genes encoding Fe-S oxidoreductases are present in $M f$. australis strain IT-1, a large number when compared to the alphaproteobacterial magnetotactic strains MC-1, AMB-1, MSR-1 and QH-2, with a single gene encoding a Fe-S oxidoreductase. The genome of $\mathrm{Ca}$. Magnetoglobus multicellularis contains 8 copies of Fe-S reductases, while strains $\mathrm{Ca}$. Magnetoovum chiemensis and $\mathrm{Ca}$. Magnetobacterium bavaricum have 10 and 13 copies, respectively. These cells synthesize a large number of magnetosomes and the presence of these reductases in greater number could be responsible for ensuring the availability of iron for cell growth and magnetosome synthesis.

\section{Carbon metabolism}

Mf. australis strain IT-1 grows chemoorganoheterotrophically on acetate and succinate. Chemolithoautotrophic growth occurs using sodium bicarbonate as the sole carbon source and thiosulfate as electron donor [13]. Genomic data suggest that autotrophic growth occurs via the reverse or reductive TCA cycle (rTCA) (Fig. 4), a trait shared with Mc. marinus strain MC-1 [10]. In other magnetotactic Alphaproteobacteria, including some Magnetospirillium species and $M v$. 
blakemorei strain MV-1 [55], autotrophic growth occurs via the Calvin-Benson-Bassham cycle. In Mf. australis strain IT-1 genome, no predicted ORF encoding the ribulose-1,5-bisphosphate carboxylase/oxygenase ( $\mathrm{Ru}$ bisCo) enzyme was detected. All enzymes required for oxidative TCA and reductive TCA cycles are encoded, but no genes encoding the enzymes necessary for the glyoxylate bypass were found.

\section{Nitrogen metabolism}

The genome of $M f$. australis strain IT-1 contains all known genes necessary for nitrogen fixation, a common trait among other magnetotactic Alphaproteobacteria, with the exception of strain QH-2 [11]. Strain IT-1 grows in medium without addition of fixed nitrogen sources and therefore, it likely is able to fix $\mathrm{N}_{2}$ under favorable environmental conditions. Genes necessary for this pathway occur in two clusters, nifZVXNEYTKDH (ORFs 03719 to 03740, with two transposases and hypothetical proteins between nif $Y$ and nifE) and nifQBA (ORFs 02324, 02328 and 02330) with genes nifR (ORF 01176) and nifU (ORF 03853) occurring elsewhere in the genome. The ammonium produced by nitrogen fixation or absorbed from the environment might be assimilated through one of three pathways for which strain IT-1 carries all the necessary genes: alanine dehydrogenase (ORF 00976), glutamate dehydrogenase (ORF 03387) or glutamine synthase and glutamate synthase (ORF 03244) (GS-GOGAT cycle). These pathways are also present in the genome of Mc. marinus strain MC-1 [10] and in other alphaproteobacterial MTB.

Several Magnetospirillum species are capable of reducing certain nitrogen oxides as terminal electron acceptors for growth, producing $\mathrm{N}_{2}$ from nitrate through denitrification [56]. The first reaction of denitrification, the reduction of $\mathrm{NO}_{3}^{-}$to $\mathrm{NO}_{2}^{-}$, is catalyzed the enzyme dissimilatory nitrate reductase, of which there are two types: a periplasmic (Nap) and a membrane-bound (Nar) form. Nap has been shown to be involved in magnetite magnetosome biomineralization, probably through redox control, in some Magnetospirillum species [57] and, interestingly, nap has been found in the genomes of all magnetotactic Alphaproteobacteria studied so far $[7,10,13]$ including strain IT1 (ORF 01154) as well as in the genome of the uncultured MTB Candidatus Magnetobacterium bavaricum from the Nitrospirae phylum [20]. All these MTB biomineralize magnetite and it is possible that Nap plays a role in the biomineralization of magnetite in all MTB that produce this mineral. Surprisingly, however despite possessing nap genes, neither $M f$. australis strain IT-1 or Mc. marinus MC-1 grows anaerobically with nitrate as a terminal electron acceptor.

The genome of Mf. australis strain IT-1 does not contain genes for the subsequent reduction steps of denitrification (nir, nor and nos genes), as do the genomes of some Magnetosprillum species and thus it appears that it is not capable of dissimilatory nitrite, nitric oxide and nitrous oxide reduction. The situation is similar with Mc. marinus strain MC-1 except that there are copies of nitric oxide reductase (norCBQ and norD) in the genome of this organism [10]. Genes for the assimilatory nitrate reduction (nas) pathway were not found in either of the genomes of $M f$. australis strain IT-1 or Mc. marinus strain MC-1 [10].

\section{Sulfur metabolism}

In aerobic environments, sulfur is mostly found in the oxidized form as sulfate $\left(\mathrm{SO}_{4}^{-2}\right)$ and must be reduced to be used by bacteria. The genome of $M f$. australis strain IT-1 contains two groups of genes involved in assimilatory sulfate reduction. Two genes responsible for assimilative sulfate reduction were found, a sulfate adenylyltransferase that turns sulfate in adenylyl sulfate and other two adenylylsulfate kinases (ORFs 01705 and 03386) (Fig. 4). Mf. australis strain IT-1 does not have genes that encode the proteins CysH and CysI, capable of catalyzing 3'-phospho- $5^{\prime}$-adenylyl sulfate to sulfite and reducing sulfite to $\mathrm{H}_{2} \mathrm{~S}$, respectively.

ORFs encoding enzymes involved in dissimilatory sulfate reduction sulfate to sulfite (dissimilatory sulfate reductase DsrAB and adenylyl-sulfate reductase - AprAB) are present in the genome of $M f$. australis strain IT-1. We found six $d s r$ genes in the $M f$. australis strain IT-1 genome, $d s r A B$ (ORFs 03242 and 03243), $d s r C$ (ORF 00945) and dsrHFE (ORFs 03513 to 03515) that are clustered close to two sulfite oxidase genes, yed $Y$ and yedZ (ORFs 03271 and 03272, respectively). This arrangement of the $d s r$ and yed genes is also similar in the genomes of Ms. magneticum strain AMB-1 and Mc. marinus strain MC-1. In some bacteria, such as Allochromatium vinosum, the Dsr (dissimilatory sulfite reductase) proteins are essential for the oxidation of zero-valent sulfur in sulfur globules [58]. We have also found a gene encoding a sulfate adenylyltransferase (ORF 02663) with higher similarity to a marine Gammaproteobacteria (accession number: WP007226305, 99 \% coverage, $78 \%$ ID and $89 \%$ positives) and genes encoding adenylylsulfate reductase subunits (AprAB, ORFs 2173 and 3566, respectively) with higher similarity to Thiocystis violascens (accession number: WP_014779579, 99 \% coverage, 67 \% ID and $77 \%$ positives) and to Acromatium sp. (accession number: KOR32136, 98 \% coverage, $85 \%$ ID and $94 \%$ positives), respectively. However, we could not unequivocally detect genes encoding the QmoABC membrane complex, responsible for electron transfer to the AprAB enzyme in sulfate reducing bacteria [59]. Under laboratory conditions, Mf. australis strain IT-1 is capable of oxidizing sulfide but does not grow anaerobically with sulfate as the sole terminal electron acceptor, however this feature would 
confer an important ecological advantage for $M f$. australis strain IT-1, since the Itaipu lagoon receives high organic matter loads that decrease $\mathrm{O}_{2}$ availability and as it is a marine habitat, the water is rich in sulfate.

The genome of $M f$. australis strain IT-1 contains two set of sox genes. Enzymes for this pathway are responsible for the oxidation of reduced sulfur compounds directly to sulfate, without a sulfite intermediate. The first set of genes is comprised of soxXYZAB (ORFs 03354 to 03358) and is organized similarly in the genome of $M c$. marinus strain MC-1. The second set consists of soxEFXY (ORFs 02177 to 02182). The soxW (ORF 01228) gene is distant from the other sox genes. Two copies of sox $Y$, that encodes an enzyme that binds the sulfur compound to be oxidized, are present (ORFs 02181 and 03355). However, soxC and soxD, both encoding for proteins involved in electron transfer chain, are not present in $M f$. australis strain IT-1. Genes encoding a sulfide quinone reductase (ORFs 01515, 02178 and 02650), a key enzyme for sulfur globule synthesis, are present in $M f$. australis strain IT-1 and confirms its ability to produce intracellular sulfur globules (Fig. 4), as observed by microscopy (see Fig. 1; [13]). Other genes encoding proteins that directly or indirectly act in sulfur metabolism are found dispersed in $M f$. australis strain IT-1 genome and include three specific sulfate transporter (ORFs 00291, 02332 and 02706), one bifunctional sulfur transporter/tiasol synthase, four sulfotransferase and seven $\mathrm{ABC}$ transporters for nitrate/sulfonate/bicarbonate.

\section{Phosphorous metabolism}

Phosphorus, generally in the form of phosphate, plays a major role in diverse cellular processes and efficient control over the uptake and storage of phosphate and the regulation of phosphate metabolism is mandatory. The genome of $M$ f. australis strain IT-1 contains genes related to the Pho regulon (two copies of phoU and phoBR, ORFs 0718, 0148; 03223 and 03224), responsible for the uptake of phosphate, as well as genes (ORFs 01828 and 03685 ) regulating the absorption of phosphonates $[60,61]$. Genes encoding the high affinity transport of phosphorus (pstBAC, ORF 04289, 04291 and 04292; pstS1 and pstS2, ORF 02354 and 03453), a permease (ORF 04293), three phosphonate transporters and a diguanylate cyclase/phosphodiesterase (ORF 04288) also occur in the genome of IT-1. A similar arrangement is present in the genome of Mc. marinus strain MC-1. This type of regulation has also been described for Magnetospirillum strains AMB-1, MS-1 and MSR-1 [10]. Both MC-1 and IT-1 have genes encoding polyphosphate kinase and exopolyphosphatase enzymes, both involved in the synthesis of polyphosphate granules [62] (Fig. 4). Polyphosphate granules function as an energy and phosphate reservoir as well as being involved in kinase reactions [63]. It has been shown that a lack of polyphosphate kinase hampers the cell response to environmental stress [64-66].

Phosphate metabolism may also be related to magnetosome biosynthesis. Phosphate granules are widespread in magnetotactic cocci whether sampled from the environment [67] or grown in culture even when the phosphate concentration is relatively low (Fig. 1, $[1,10])$. It has been shown the involvement of a phosphate-rich ferric hydroxide phase for storage of iron inside magnetosome vesicle before magnetite crystals formation [68].

\section{Detoxification of reactive oxygen species}

The genome of $M f$. australis strain IT- 1 contains 9 ORFs encoding proteins involved in detoxifying reactive oxygen species. There are four ORFs encoding cytochrome C peroxidase (ORFs 00488, 02063, 03177 and 03947), three encoding alkyl hydroperoxide reductase (ORFs 02341, 02949 and 03103), one catalase (ORF 01369) and one superoxide dismutase (02343). This number of ORFs is lower than that encoded in the genome of Ms. magneticum strain AMB-1 (15) but much higher than the 3 genes encountered in the Mc. marinus strain MC- 1 genome, implying that $M f$. australis might be more resistant to oxidative stress than the marine magnetotactic cocci.

\section{Conclusions}

The characterization of MTB, whether cultured or uncultured, is largely based on morphological, behavioral, metabolic and genomic aspects. Here we used phenotypic and genotypic data to describe features in $M f$. australis strain IT-1, a cultured magnetotactic coccus, associated with its metabolism, its ability to produce magnetosomes, and its structure and behavior. The unique alignment of the linear magnetosome chain with flagella bundles may indicate the rotating cell body observed associated with a high speed swimming may help $M f$. australis strain IT- 1 to overcome physical barriers of objects encountered in sediment or help to quickly move away detrimental stimuli. Environmental conditions in Itaipu lagoon change constantly and the high number of genes encoding transport and transduction genes in strain IT-1 favors its survival. Genomic analysis indicates Mf. australis strain IT-1 and Mc. marinus strain MC-1 are closely related species but contain some different, distinctive features. Similarity values between the amino acid sequences of homologous proteins are not very high and genomic sequencing of other magnetotactic cocci such the marine bacterium strain MO-1 will improve our knowledge regarding the magnetotactic cocci in general based on genomic analyses.

\section{Methods}

Mf. australis strain IT-1 was routinely cultured in a semisolid, heterotrophic medium [13]. High-speed 
imaging was done on a Nikon Eclipse Ti microscope equipped with a CFI Plan Apo 100x/1.45 objective lens and attached to a Photron Model 675K-M1 Fastcam camera (Photron, San Diego, CA). Images were analyzed using the free ImageJ software (rsb.info.nih.gov/ij/). Scanning electron microscopy was performed as described in [69]. For high pressure freezing and freeze substitution, a culture of $M f$. australis strain IT-1 was diluted in artificial seawater and magnetically concentrated. For freeze substitution, samples were quick-frozen with a Leica HPM 100 high pressure freezing apparatus (Leica Microsystems, Bannockburn, IL, USA) and transferred to a fixative solution containing $2 \%$ osmium tetroxide and $0.1 \%$ uranyl acetate in anhydrous acetone. Samples were kept at $-90{ }^{\circ} \mathrm{C}$ for $90 \mathrm{~h}$, then at $-35{ }^{\circ} \mathrm{C}$ for $4 \mathrm{~h}$ and $-20{ }^{\circ} \mathrm{C}$ for $2 \mathrm{~h}$. The temperature was gradually increased to room temperature and samples were embedded and polymerized in PolyBed 812. Ultrathin sections were obtained on Leica EM U6 ultramicrotome (Leica Microsystems, Bannockburn, IL, USA), stained with uranyl acetate and lead citrate and imaged with a Morgagni transmission electron microscope (FEI Company, Hillsboro, OR, USA) at 80kV. Electron tomography was performed using a JEOL JEM 2100F transmission electron microscope operated at $200 \mathrm{kV}$ using STEM/HAADF mode. Tomographic tilt series were acquired by tilting the samples from at least $-60^{\circ}$ to $+60^{\circ}$ at $1.46^{\circ}$ intervals. Fast Fourier Transform (FFT) was obtained from high-resolution calibrated images using Digital Micrograph software (Gatan Inc.). 3D models of the crystals were constructed using KrystalShaper program (JCrystalSoft). Electron tomography was aligned and reconstructed using software Image (http://rsb.info.nih.gov/ij/) with the plug-in TomoJ. 3D model was obtained using UCSF Chimera software (University of California, San Francisco, http://www.cgl.ucsf.edu/chimera/).

For DNA preparation and pyrosequencing, cells were concentrated by centrifugation and washed several times with sterile distilled water. DNA was sequenced on a 454 GS FLX System sequencer (Roche Diagnostics GmbH/454 Life Sciences Corporation, Branford, CT, USA) and the assembly was performed using WGS-Assembler v7.0 and Newbler $\mathrm{v} 2.8$, and the contigs were joined with homemade scripts. The genome has been analyzed with SABIA (System for Automated Bacterial Integrated Annotation) platform [70]. Gene prediction was obtained with the Glimmer and genes were annotated using blast (query and subject coverage equal to $60 \%$ and positives equal $50 \%$ ) and KEGG database. Mam genes synteny were analyzed based on the Bidirectional Best Hit (BBH) approach using BLASTP Program. All BLASTP searches were performed with an e-value equal 1e-05, query coverage equal $60 \%$ and similarity equal $50 \%$ [40].

\section{Additional files} Additional file 1: Magnetotactic bacteria genomes (March 2016) [6-22].
(DOCX $15 \mathrm{~kb}$ )

Additional file 2: General information on Mf. australis strain IT-1 genome sequence. (DOCX $12 \mathrm{~kb}$ )

Additional file 3: Comparison of Mf. australis contig containing mam genes and the putative MAl of Mc. marinus. Schematic comparison of Mf. australis strain IT-1 contig containing mam genes and its corresponding region in the putative $\mathrm{MAI}$ of $\mathrm{Mc}$. marinus strain $\mathrm{MC}-1$. Similar regions are marked in boxes (pink, yellow, blue and gray). Grey ORFs represent those that encode non-hypothetical proteins. Annotation was omitted at the end of the putative MAI of Mc. marinus strain MC- 1 because it does not correspond to any region of $M f$. australis strain IT-1 present above. tnp: transposase; rsl: resolvase; HP: hypothetical protein; aa carrier: amino acid carrier protein; mcp: methyl-accepting chemotaxis protein; hm:

hemerythrin-like protein; ft: ferritin-like protein; fis: Fis family transcriptional regulator; int: integrase; par: chromosome partitioning protein; trfA: replication initiator protein A. Unique Mf. australis strain IT1 hypothetical proteins are labeled with asterisks. (PNG 365 kb)

Additional file 4: Classes of MCPs annotated in Mf. australis. Conserved sensory or ligand binding regions are presented. (DOCX $12 \mathrm{~kb}$ )

Additional file 5: Genes involved in signal transduction in Mf. australis strain IT-1. (DOCX 25 kb)

\section{Acknowledgements}

We thank Dr. Juliana Lopes from INMETRO, Brazil for help with freezesubstitution and Dr. Bechara Kachar from Laboratory of Cell Structure and Dynamics, NIDCD, NIH, USA for high speed imaging. We thank Dr. Marcos Farina from ICB-UFRJ and Brazilian Center for Physics Research (CBPF) for HRTEM facilities

\section{Declarations}

About this supplement

This article has been published as part of BMC Genomics Volume 17 Supplement 8: Selected articles from the Sixth International Conference of the Iberoamerican Society for Bioinformatics on Bioinformatics and Computational Biology for Innovative Genomics. The full contents of the supplement are available online at https://bmcgenomics.biomedcentral.com/ articles/supplements/volume-17-supplement-8.

\section{Funding}

This work was funded by Brazilian CAPES, FAPERJ and CNPq. DAB is supported by U.S. National Science Foundation (NSF) grant EAR-1423939. The publication charges for this article were funded by Brazilian FAPERJ (Fundação de Amparo à Pesquisa do Estado do Rio de Janeiro) grant number E26/203.038/2015"

\section{Availability of data and materials}

This Whole Genome Shotgun project has been deposited at DDBJ/ENA/ GenBank under the accession LVJN00000000. The version described in this paper is version LVJN01000000.

\section{Authors' contribution}

ACVA analyzed genomic data and wrote the manuscript. VM analyzed genomic data, processed and analyzed microscopy experiments. JC performed crystallographic characterization and contributed to the manuscript. LCRST, PL and SL contributed data and performed genomic annotation. LGA and ATRV provided bioinformatics resources. FA analyzed genomic data and wrote the manuscript. UL processed samples, acquired high-speed imaging and electron microscopy data. UL, FA and DAB designed and coordinated the study, and wrote the manuscript. All authors gave final approval for publication.

\section{Competing interests}

The authors declare that they have no competing interests.

Consent for publication

Not applicable. 


\section{Ethics approval and consent to participate}

Not applicable.

\begin{abstract}
Author details
'Instituto de Microbiologia Paulo de Góes, Universidade Federal do Rio de Janeiro, 21941-902 Rio de Janeiro, RJ, Brazil. ${ }^{2}$ School of Life Sciences, University of Nevada at Las Vegas, Las Vegas, NV 89154-4004, USA. ${ }^{3}$ Departamento de Matemática Aplicada e Computacional, Laboratório Nacional de Computação Científica, 25651-070 Petrópolis, RJ, Brazil. ${ }^{4}$ Current institution: Departamento de Biologia, Universidade Federal de São Carlos, 18052-780 Sorocaba, SP, Brazil.
\end{abstract}

\section{Published: 25 October 2016}

\section{References}

1. Bazylinski DA, Frankel RB. Magnetosome formation in prokaryotes. Nat Rev Microbiol. 2004;2:217-30

2. Flies CB, Peplies J, Schüler D. Combined approach for characterization of uncultivated magnetotactic bacteria from various aquatic environments. Appl Environ Microb. 2005;71:2723-31.

3. Lin W, Pan Y. Uncultivated magnetotactic cocci from Yuandadu park in Beijing, China. Appl Environ Microb. 2009;75:4046-52.

4. Lin W, Li J, Pan Y. Newly isolated but uncultivated magnetotactic bacterium of the phylum Nitrospirae from Beijing, China. Appl Environ Microb. 2012;78:668-75.

5. Frankel RB, Bazylinski DA, Johnson MS, Taylor BL. Magneto-aerotaxis in marine coccoid bacteria. Biophys J. 1997;73:994-1000.

6. Wang X, Wang Q, Zhang W, Wang Y, Li L, Wen T, et al. Complete genome sequence of Magnetospirillum gryphiswaldense MSR-1. Genome Announc. 2014. doi:10.1128/genomeA.00171-14.

7. Matsunaga T, Okamura Y, Fukuda Y, Wahyudi AT, Murase Y, Takeyama H. Complete genome sequence of the facultative anaerobic magnetotactic bacterium Magnetospirillum sp. strain AMB-1. DNA Res. 2005;12:157-66.

8. Smalley MD, Marinov GK, Bertani LE, DeSalvo G. Genome sequence of Magnetospirillum magnetotacticum strain MS-1. Genome Announc. 2015;3: e00233-15.

9. Grouzdev DS, Dziuba MV, Sukhacheva MS, Mardanov AV, Beletskiy AV, Kuznetsov BB, et al. Draft genome sequence of Magnetospirillum sp. strain SO-1, a freshwater magnetotactic bacterium isolated from the Ol'khovka River, Russia. Genome Announc. 2014;2:e00235-14.

10. Schübbe S, Williams TJ, Xie G, Kiss HE, Brettin TS, Martinez D, et al. Complete genome sequence of the chemolithoautotrophic marine magnetotactic coccus strain MC-1. App Environ Microbiol. 2009;75:4835-52.

11. Ji B, Zhang SD, Arnoux P, Rouy Z, Alberto F, Philippe N, et al. Comparative genomic analysis provides insights into the evolution and niche adaptation of marine Magnetospira sp. QH-2 strain. Environ Microbiol. 2014;16:1-20.

12. Nakazawa H, Arakaki A, Narita-Yamada S, Yashiro I, Jinno K, Aoki N, et al. Whole genome sequence of Desulfovibrio magneticus strain RS-1 revealed common gene clusters in magnetotactic bacteria. Genome Res. 2009;19:1801-8.

13. Morillo V, Abreu F, Araujo AC, de Almeida LGP, Enrich-Prast A, Farina M, et al. Isolation, cultivation and genomic analysis of magnetosome biomineralization genes of a new genus of South-seeking magnetotactic cocci within the Alphaproteobacteria. Front Microbiol. 2014;5:1-12. doi:10. 3389/fmicb.2014.00072.

14. Jogler C, Kube M, Schübbe S, Ullrich S, Teeling H, Bazylinski DA, et al. Comparative analysis of magnetosome gene clusters in magnetotactic bacteria provides further evidence for horizontal gene transfer. Environ Microbiol. 2009;11:1267-77.

15. Abreu F, Morillo V, Nascimento FF, Werneck C, Cantão ME, Ciapina LP, et al. Deciphering unusual uncultured magnetotactic multicellular prokaryotes through genomics. ISME J. 2014;8:1055-68.

16. Kolinko S, Richter M, Glöckner FO, Brachmann A, Schüler D. Single-cell genomics reveals potential for magnetite and greigite biomineralization in an uncultivated multicellular magnetotactic prokaryote. Environ Microbiol Rep. 2012;6:524-31.

17. Lefèvre $C T$, Menguy $N$, Abreu F, Lins U, Pósfai M, Prozorov T, et al. A cultured greigite-producing magnetotactic bacterium in a novel group of sulfate-reducing bacteria. Science. 2011;334:1720-3.

18. Lefèvre $C T$, Trubitsyn $D$, Abreu F, Kolinko $S$, Jogler $C$, de Almeida $L G$, et al. Comparative genomic analysis of magnetotactic bacteria from the Deltaproteobacteria provides new insights into magnetite and greigite magnetosome genes required for magnetotaxis. Environ Microbiol. 2013;15:2712-35.

19. Kolinko S, Richter M, Glöckner FO, Brachmann A, Schüler D. Single-cell genomics of uncultivated deep-branching magnetotactic bacteria reveals a conserved set of magnetosome genes. Environ Microbiol. 2016;18:21-37.

20. Lin W, Deng A, Wang Z, Li Y, Wen T, Wu LF, et al. Genomic insights into the uncultured genus 'Candidatus Magnetobacterium' in the phylum Nitrospirae. ISME J. 2014;8:2463-77.

21. Rinke C, Schwientek P, Sczyrba A, Ivanova NN, Anderson IJ, Cheng JF, et al. Insights into the phylogeny and coding potential of microbial dark matter. Nature. 2013;499:431-7.

22. Lin W, Pan Y. A putative greigite-type magnetosome gene cluster from the candidate phylum Latescibacteria. Environ Microbiol Rep. 2015;7:237-42.

23. Blakemore RP, Frankel RB, Kalmijn AJ. South-seeking magnetotactic bacteria in the southern-hemisphere. Nature. 1980;286:384-5.

24. Lins U, Farina M. Magnetosome chain arrangement and stability in magnetotactic cocci. Antonie Van Leeuwenhoek. 2004;85:335-41.

25. Katzmann E, Scheffel A, Gruska M, Plitzko JM, Schüler D. Loss of the actin-like protein MamK has pleiotropic effects on magnetosome formation and chain assembly in Magnetospirillum gryphiswaldense. Mol Microbiol. 2010;77:208-24.

26. Abreu F, Sousa AA, Aronova MA, Kim Y, Cox D, Leapman RD, et al. Cryoelectron tomography of the magnetotactic vibrio Magnetovibrio blakemorei: Insights into the biomineralization of prismatic magnetosomes. J Struct Biol. 2013:181:162-8.

27. Briegel A, Ortega DR, Tocheva El, Wuichet K, Li Z, Chen S, et al. Universal architecture of bacterial chemoreceptor arrays. Proc Natl Acad Sci U S A. 2009;106:17181-6.

28. Zhang WJ, Santini CL, Bernadac A, Ruan J, Zhang SD, Kato T, et al. Complex spatial organization and flagellin composition of flagellar propeller from marine magnetotactic ovoid strain MO-1. J Mol Biol. 2012;416:558-70.

29. Zhang WY, Zhou K, Pan HM, Yue HD, Jiang M, Xiao T, et al. Two genera of magnetococci with bean-like morphology from intertidal sediments of the Yellow Sea, China. Appl Environ Microbiol. 2012;78:5606-11.

30. Lefèvre CT, Bennet M, Landau L, Vach P, Pignol D, Bazylinski DA, et al. Diversity of magneto-aerotactic behaviors and oxygen sensing mechanisms in cultured magnetotactic bacteria. Biophysical J. 2014;107:527-38.

31. Ruan J, Kato T, Santini CL, Miyata T, Kawamoto A, Zhang WJ, et al. Architecture of a flagellar apparatus in the fast-swimming magnetotactic bacterium MO-1. Proc Natl Acad Sci U S A. 2012;109:20643-8.

32. Zhang SD, Petersen N, Zhang WJ, Cargou S, Ruan J, Murat D, et al. Swimming behavior and magnetotaxis function of the marine bacterium strain MO-1. Environ Microbiol Rep. 2014;6:14-20.

33. Lefèvre CT, Bernadac A, Yu-Zhang K, Pradel N, Wu LF. Isolation and characterization of a magnetotactic bacterial culture from the Mediterranean Sea. Environ Microbiol. 2009;11:1646-57.

34. Lefèvre CT, Santini CL, Bernadac A, Zhang W, Li Y, Wu LF. Calcium ionmediated assembly and function of glycosylated flagellar sheath of marine magnetotactic bacterium. Mol Microbiol. 2010;78:1304-12.

35. Freitas F, Keim CN, Kachar B, Farina M, Lins U. Envelope ultrastructure of uncultured naturally occurring magnetotactic cocci. FEMS Microbiol Lett. 2003;219:33-8

36. Philippe N, Wu LF. An MCP-Like protein interacts with the MamK cytoskeleton and is involved in magnetotaxis in Magnetospirillum magneticum AMB-1. J Mol Biol. 2010;400:309-22.

37. Lacal J, García-Fontana C, Muñoz-Martínez F, Ramos JL, Krell T. Sensing of environmental signals: classification of chemoreceptors according to the size of their ligand binding regions. Environ Microbiol. 2010;12:2873-84.

38. Pósfai M, Lefèvre CT, Trubitsyn D, Bazylinski DA, Frankel RB. Phylogenetic significance of composition and crystal morphology of magnetosome minerals. Front Microbiol. 2013;4:344. doi:10.3389/fmicb.2013.00344.

39. Paul K, Nieto VM, Carlquist W, Blair DF, Harshey RM. The c-di-GMP binding protein YcgR controls flagellar motor direction and speed to affect chemotaxis by a "backstop brake" mechanism. Mol Cell. 2010;38:128-39.

40. Altschul SF, Gish W, Miller W, Myers EW, Lipman DJ. Basic local alignment search tool. J Mol Biol. 1990;215:403-10.

41. Wadhams GH, Armitage JP. Making sense of it all: bacterial chemotaxis. Nature Reviews Mol Cell Bio. 2004;5:1024-37.

42. Wuichet $\mathrm{K}$, Alexander RP, Zhulin IB. Comparative genomic and protein sequence analyses of a complex system controlling bacterial chemotaxis. Methods Enzymol. 2007:422:1-31. 
43. Letunic I, Doerks T, Bork P. SMART: recent updates, new developments and status in 2015. Nucl Acids Res. 2015;43:257-60.

44. Marchler-Bauer A, Derbyshire MK, Gonzales NR, Lu S, Chitsaz F, Geer LY, et al. CDD: NCBI's conserved domain database. Nucleic Acids Res. 2015; D222-6. doi:10.1093/nar/gku1221.

45. Bardwell L, Zou X, Nie Q, Komarova NL. Mathematical models of specificity in cell signaling. Biophys J. 2007;92:3425-41.

46. French CE, Bell JML, Ward BF. Diversity and distribution of hemerythrin-like proteins in prokaryotes. FEMS Microbiol Lett. 2008;279:131-45.

47. Komeili A. Molecular Mechanisms of Compartmentalization and Biomineralization in Magnetotactic Bacteria. FEMS Microbiol Rev. 2012;36:232-55.

48. Uebe R, Voigt B, Schweder T, Albrecht D, Katzmann E, Lang C, et al. Deletion of a fur-like gene affects iron homeostasis and magnetosome formation in Magnetospirillum gryphiswaldense. J Bacteriol. 2010;192:4192-204.

49. Rong C, Zhang C, Zhang Y, Qi L, Yang J, Guan G, et al. FeoB2 Functions in Magnetosome Formation and Oxidative Stress Protection in Magnetospirillum gryphiswaldense Strain MSR-1. J Bacteriol. 2012;194:3972-6.

50. Taabazuing CY, Hangasky JA, Knapp M. Oxygen sensing strategies in mammals and bacteria. J Inorg Biochem. 2014;133:63-72.

51. Andrews S. The Ferritin-like superfamily: Evolution of the biological iron storeman from a rubrerythrin-like ancestor. Biochim Biophys Acta. 2010; 1800:691-705

52. Lefèvre $C T$, Trubitsyn D, Abreu F, Kolinko S, Almeida LGP, Vasconcelos ATR et al. Monophyletic origin of magnetotaxis and the first magnetosomes. Environ Microbiol. 2013;15:2267-74

53. Hantke K. Iron and metal regulation in bacteria. Curr Opin Microbiol. 2001:4:172-7

54. Qi L, Li J, Zhang W, Liu J, Rong C, Li Y, et al. Fur in Magnetospirillum gryphiswaldense influences magnetosomes formation and directly regulates the genes involved in iron and oxygen metabolism. PLoS One. 2012;7: e29572. doi:10.1371/journal.pone.0029572.

55. Bazylinski DA, Dean AJ, Williams TJ, Long LK, Middleton SL, Dubbels BL. Chemolithoautotrophy in the marine, magnetotactic bacterial strains MV-1 and MV-2. Arch Microbiol. 2004;182:373-87.

56. Bazylinski DA, Blakemore RP. Denitrification and assimilatory nitrate reduction in Aquaspirillum magnetotacticum. Appl Environ Microbiol. 1983; 46:1118-24.

57. Li Y, Katzmann E, Borg S, Schüler D. The periplasmic nitrate reductase Nap is required for anaerobic growth and involved in redox control of magnetite niomineralization in Magnetospirillum gryphiswaldense. J Bacteriol. 2012;194: 4847-56.

58. Eisen JA, Nelson KE, Paulsen IT, Heidelberg JF, Wu M, Dodson RJ, et al. The complete genome sequence of Chlorobium tepidum TLS, a photosynthetic, anaerobic, green-sulfur bacterium. Proc Natl Acad Sci U S A. 2002:99:9509-14.

59. Duarte $A G$, Santos AA, Pereira IAC. Electron transfer between the QmoABC membrane complex and adenosine 5'-phosphosulfate reductase. Biochim Biophys Acta. 2016;1857:380-6.

60. Wanner BL, Chang BD. The phoBR operon in Escherichia coli K-12. J Bacteriol. 1987;169:5569-74.

61. Santos-Beneit $F$. The Pho regulon: a huge regulatory network in bacteria Front Microbiol. 2015;6:402. doi:10.3389/fmicb.2015.00402.

62. Ahn K, Kornberg A. Polyphosphate kinase from Escherichia coli. Purification and demonstration of a phosphoenzyme intermediate. J Biol Chem. 1990; 265:11734-9.

63. Kornberg A. Inorganic Polyphosphate: Toward Making a Forgotten Polymer Unforgettable. J Bacteriol. 1995;177:491-6.

64. Rashid MH, Rumbaugh K, Passador L, Davies DG, Hamood AN, Iglewski BH, et al. Polyphosphate kinase is essential for biofilm development, quorum sensing, and virulence of Pseudomonas aeruginosa. Proc Natl Acad Sci U S A. 2000;97:9636-41.

65. Jahid IK, Silva AJ, Benitez JA. Polyphosphate stores enhance the ability of Vibrio cholerae to overcome environmental stresses in a low-phosphate environment. Appl Environ Microbiol. 2006;72:7043-9.

66. Lindner SN, Knebel S, Wesseling H, Schoberth SM, Wendisch VF. Exopolyphosphatases PPX1 and PPX2 from Corynebacterium glutamicum. Appl Environ Microbiol. 2009;75:3161-70.

67. Lins $U$, Farina M. Phosphorus-rich granules in uncultured magnetotactic bacteria. FEMS Microbiol Lett. 1999;172:23-8.

68. Baumgartner J, Morin G, Menguy N, Gonzalez TP, Widdrat M, Cosmidis J, et al. Magnetotactic bacteria form magnetite from a phosphate-rich ferric hydroxide via nanometric ferric (oxyhydr)oxide intermediates. Proc Natl Acad Sci U S A. 2013;110:14883-8.

69. Keim CN, Martins JL, Abreu F, Rosado AS, de Barros HL, Borojevic R, et al. Multicellular life cycle of magnetotactic prokaryotes. FEMS Microbiol Lett. 2004;240:203-8.

70. Almeida LG, Paixão R, Souza RC, Da Costa GC, Barrientos FJ, Dos Santos MT, et al. A system for automated bacterial (genome) integrated annotation-SABIA. Bioinformatics. 2004;20:2832-3.

\section{Submit your next manuscript to BioMed Central and we will help you at every step:}

- We accept pre-submission inquiries

- Our selector tool helps you to find the most relevant journal

- We provide round the clock customer support

- Convenient online submission

- Thorough peer review

- Inclusion in PubMed and all major indexing services

- Maximum visibility for your research

Submit your manuscript at www.biomedcentral.com/submit
Biomed Central 